\title{
Margin Uninvolved by Invasive Carcinoma
}

National Cancer Institute

\section{Source}

National Cancer Institute. Margin Uninvolved by Invasive Carcinoma. NCI Thesaurus.

Code C159234.

A result that the marg in is not uninvolved by invasive carcinoma. 